\title{
Securing Babies" Access to Life-Saving Medicine
}

Susan Hepworth

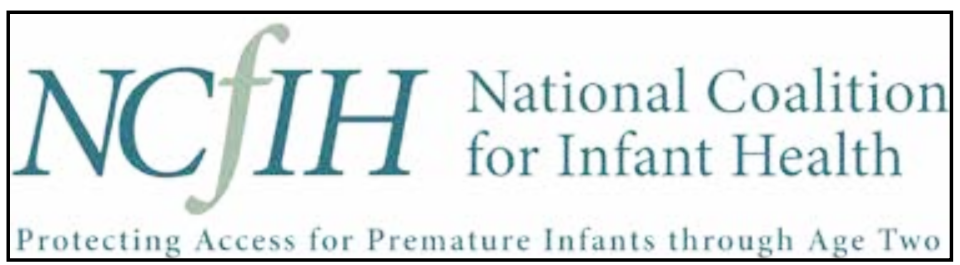

The National Coalition for Infant Health is a collaborative of more than 200 professional, clinical, community health, and family support organizations focused on improving the lives of premature infants through age two and their families. NCfIH's mission is to promote lifelong clinical, health, education, and supportive services needed by premature infants and their families. NCfIH prioritizes safety of this vulnerable population and access to approved therapies.

Bringing a newborn baby home from the hospital is a joy like no other. Bringing that same baby back to the hospital for treatment of a life-threatening virus would be a nightmare. Yet, for tens of thousands of parents, $\underline{\mathrm{RSV}}$ makes that traumatic return trip a reality. (1)

While there is no vaccine to prevent the respiratory syncytial virus, "vaccine-like" drugs can protect babies through their most vulnerable years. These drugs, called monoclonal antibodies, deliver protective antibodies by enlisting the body's natural immune system response against infection like RSV. Realizing their potential for all babies means improving their accessibility. That comes down to the question of whether policymakers and insurance companies will cover these medications as vaccines.

If they do, the drugs might be eligible for the national Vaccines for Children Program (VCP). (2) VCP would expand the num- ber of protected children and reduce disparities in access by providing vaccine-like drugs to eligible children at no cost. Such an approach would benefit children insured by Medicaid as well as those who are under or uninsured.

By contrast, if vaccine-like products are treated as traditional medications, they will be more costly for parents and likely harder to get. Most commercial health plans require patients to pay at least a flat co-pay for prescriptions, and uninsured patients are usually responsible for a medication's total cost. It is also common for insurers to limit access through the use of prior authorization. (3) Out-of-pocket costs and insurance barriers will keep kids from reaping the benefits of these preventive medications.

To understand why vaccine-like medications need to be widely available, consider the scope of RSV in America.

\section{"Vaccine-like medications are a welcome tool to protect infants and their families from the torment of a serious RSV infection. Treating these drugs like vaccines will assure more equitable access and save the high costs of treating preventable infections."}

It is one of the most common causes of lower respiratory tract infections in young children and the leading cause of hospitalization (4) among babies less than one year old. The majority of RSV hospitalizations are in healthy infants born at full term.

Most RSV infections produce cold-like symptoms and are easily treated. But the virus can be much more dangerous in some in-

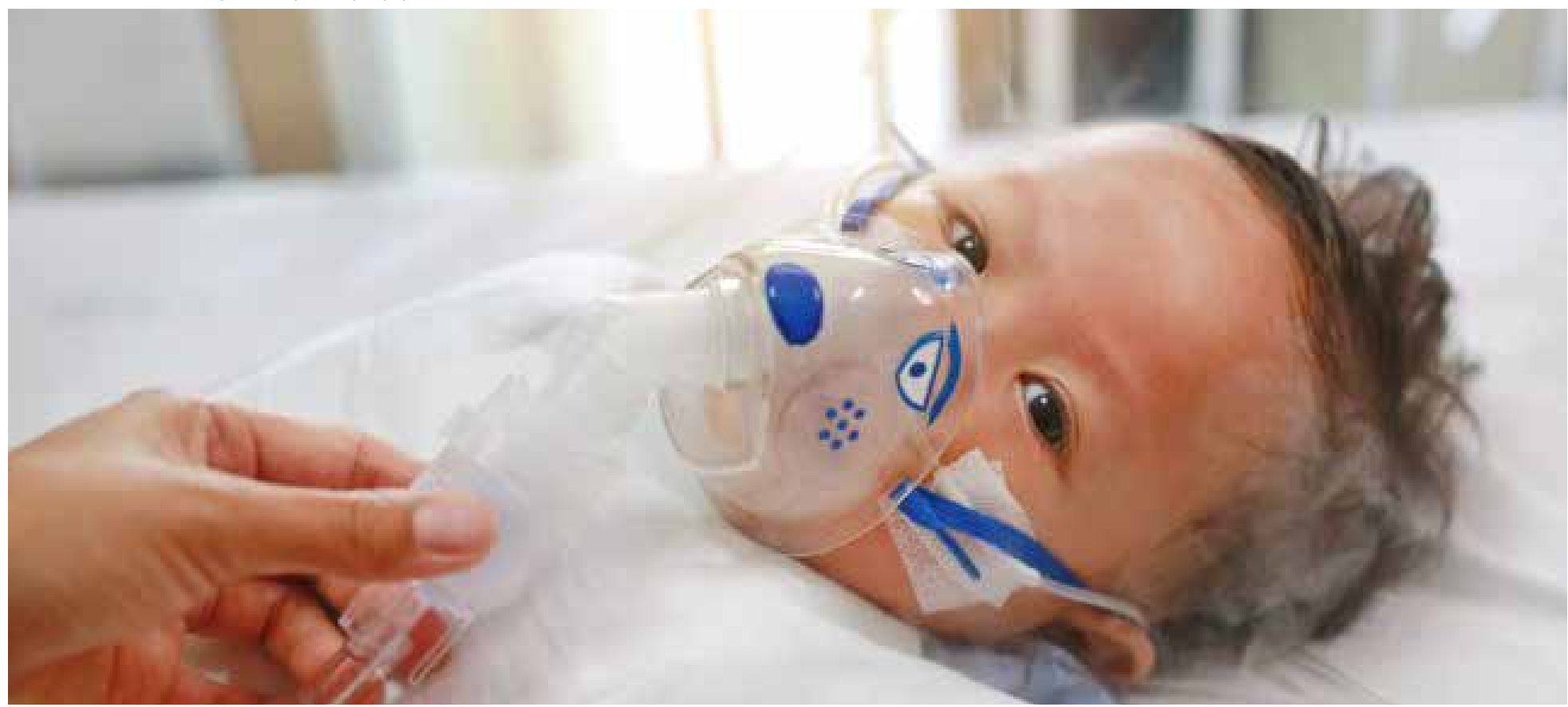


fants and children. Research cites a greater rate of serious cases in babies from lower socioeconomic groups. (4)

Vaccine-like medications are a welcome tool to protect infants and their families from the torment of a serious RSV infection. Treating these drugs like vaccines will assure more equitable access and save the high costs of treating preventable infections. The net result is lower costs and healthier kids.

\section{References:}

1. https://www.cdc.gov/rsv/high-risk/infants-young-children. html

2. https://www.cdc.gov/vaccines/programs/vfc/index.html

3. $\quad$ https://www. youtube.com/watch? v=aibWAFpkht0\&t=15s

4. https://www.ncbi.n/m.nih.gov/pmc/articles/ PMC5833316/\#: :text=Palivizumab\%20is\%20a\%20monoclonal\%20antibody, infections $\% 20$ in $\% 20$ high $\% 2$ Drisk $\% 20$ infants.

Disclosure: The author has no relevant disclosures.

\section{NT}

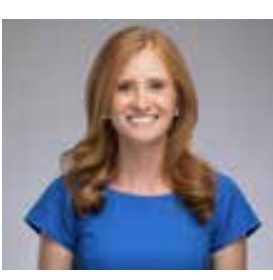

Susan Hepworth

Director

National Coalition for Infant Health

1275 Pennsylvania Ave. NW, Suite 1100A

Washington, DC 20004

Email: info@infanthealth.org

\section{OPIOIDS and NAS}

When reporting on mothers, babies,

and substance use

\section{LANGUAGE MATTERS}

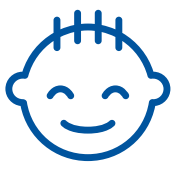

I am not an addict.

I was exposed to substances in utero. I am not addicted. Addiction is a set of behaviors associated with having a Substance Use Disorder (SUD).

\section{I was exposed to opioids.}

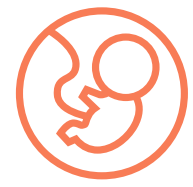

While I was in the womb my mother and I shared a blood supply. I was exposed to the medications and substances she used. I may have become physiologically dependent on some of those substances.

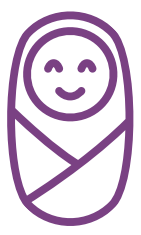

\section{NAS is a temporary and treatable condition.}

There are evidence-based pharmacological and non-pharmacological treatments for Neonatal Abstinence Syndrome.

\section{My mother may have a SUD.}

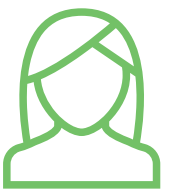

She might be receiving Medication-Assisted Treatment (MAT). My NAS may be a side effect of her appropriate medical care. It is not evidence of abuse or mistreatment.

\section{My potential is limitless.}

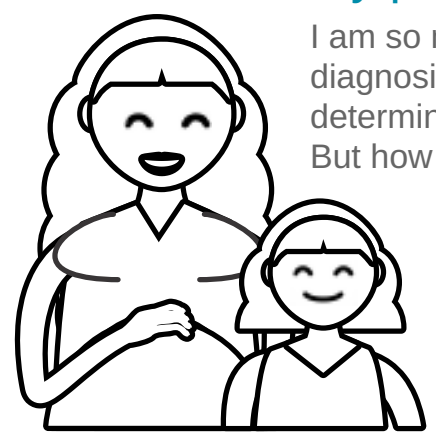

am so much more than my NAS diagnosis. My drug exposure will not my long-term outcomes. you treat me will. When you invest in my family's health and wellbeing by supporting Medicaid and Early Childhood Education you can expect that I will do as well as any of my peers!
Learn more about

Neonatal Abstinence Syndrome at www.nationalperinatal.org

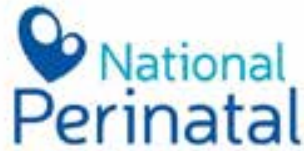

Association Restrictions on care and treatment should not worsen inherent disparities. 